ORIGINAL

\title{
Supervivencia de postlarvas de camarón blanco Litopenaeus vannamei a diferentes salinidades y temperaturas
}

\section{Survival of white shrimp Litopenaeus vannamei poslarvae at different salinities and temperatures}

\author{
Juan Arzola G, ${ }^{1}$ M.Sc, Pablo Piña $\mathrm{V}^{1,2 *}$ Ph.D, Mario Nieves $\mathrm{S}^{1,2}$ Ph.D, \\ María Medina J, 2 M.Sc.
}

\begin{abstract}
${ }^{1}$ Universidad Autónoma de Sinaloa, Facultad de Ciencias Químico Biológicas, Programa Regional del Noroeste para el Doctorado en Biotecnología, Av. De las Américas y Blvd. Universitarios s/n. Ap. Postal 1354. Culiacán, Sinaloa, México. Tel/Fax: 66771378 60. ${ }^{2}$ Universidad Autónoma de Sinaloa, Facultad de Ciencias del Mar, Paseo Claussen s/n. Ap. Postal 610. Mazatlán, Sinaloa, México. *Correspondencia: pablopina@live.com.mx
\end{abstract}

Recibido: Noviembre de 2012; Aceptado: Febrero de 2013.

\section{RESUMEN}

Objetivo. Analizar el efecto combinado de salinidad $(5,15,25,35$ y 45 ups) y temperatura $(15,20$, 25,30 y $35^{\circ} \mathrm{C}$ ) sobre la supervivencia y frecuencia de mudas en postlarvas (PL12) de Litopenaeus vannamei. Materiales y métodos. Los experimentos se realizaron por cuadriplicado por cada combinación de salinidad y temperatura. Las postlarvas se mantuvieron en acuarios de $3 \mathrm{~L}$ a una densidad de 100PL/I. La salinidad se incrementó disolviendo sal granulada libre de yodo, a partir de agua de mar, mientras que para alcanzar las salinidades menores se utilizó agua dulce filtrada. Las postlarvas aclimatadas a 35 ups y $28^{\circ} \mathrm{C}$ (condición inicial) fueron sometidas abruptamente a las salinidades y temperaturas experimentales por 30 minutos. Después, se filtraron y se colocaron nuevamente en su condición inicial para su recuperación. Transcurridos 30 minutos, se contaron las postlarvas vivas y las mudas. Los datos fueron analizados por un ANOVA de dos vías. Resultados. Se determinó que la supervivencia y frecuencia de mudas fueron significativamente afectadas por la salinidad, temperatura y su interacción $(p<0.05)$. Se obtuvo una supervivencia máxima y mínima de 99.8 y $94.4 \%$. Los porcentajes de supervivencia y frecuencias de mudas disminuyeron a una baja salinidad en combinación a bajas y altas temperaturas, y aumentaron independientemente de la salinidad y temperatura, a excepción de 5 ups con 15,30 y $35^{\circ} \mathrm{C}$. La mortalidad máxima de postlarvas fue de $5.6 \%$ ( 5 ups y $30^{\circ} \mathrm{C}$ ). Conclusiones. Se determinó que a temperaturas de 20 y $25^{\circ} \mathrm{C}$ en combinación a las salinidades utilizadas, fueron las mejores condiciones de supervivencia y de resistencia de las postlarvas ante dichas condiciones hidrológicas.

Palabras clave: Aclimatación, ambiente acuático, salinidad del agua (Fuente: DeCS). 


\section{ABSTRACT}

Objective. The combined effect of salinity $(5,15,25,35$, and $45 \mathrm{psu})$ and temperature $(15,20,25,30$, and $35^{\circ} \mathrm{C}$ ) was evaluated on survival and molt frequency in Litopenaeus vannamei postlarvae (PL12). Materials and methods. Four repetitions were applied to each combination of salinity and temperature. The PL were placed in $3 \mathrm{~L}$ beakers at a density of $100 \mathrm{PL} / \mathrm{l}$. Salinity was increased by dissolving commercial salt without iodine, into marine water, whereas fresh filtered tap water was used to decrease the salinity from seawater. The PL were adapted at $35 \mathrm{psu}$ and $28^{\circ} \mathrm{C}$ (initial conditions) during 30 minutes. Thereafter, they were transferred to each experimental combination of salinity and temperature by 30 minutes. Following this procedure, the PL were filtered and retuned to their initial acclimatization conditions. After 30 minutes of recuperation, the live PL and molts were counted. All data were analyzed using a two-way ANOVA. Results. Survival and molt frequency were significantly $(p<0.05)$ affected by salinity, temperature and the interaction of both factors. The maximum and minimum survival rates of 99.8 and $94.4 \%$ were obtained. Survival and molt frequency rates were significantly $(p<0.05)$ affected by salinity, temperature and the interaction of both factors. Survival and molt frequency percentage rates decreased at lower salinity in combination with lowest and highest temperatures, and significantly increased, independently from salinity and temperature conditions, with exception of $5 \mathrm{psu}$ at 15,30 and $35^{\circ} \mathrm{C}$. The highest mortality rate $(5.6 \%)$ was obtained at $5 \mathrm{psu}$ and $30^{\circ} \mathrm{C}$. Conclusions. It was determined that survival of postlarvae was higher at temperatures of 20 and $25^{\circ} \mathrm{C}$ given salinity combination, which are considered the best conditions for survival and resistance.

Key words: Acclimatization, aquatic environment, saline waters (Source: DeCS).

\section{INTRODUCCIÓN}

El camarón blanco Litopenaeus vananmei es una especie comúnmente cultivada en el noroeste de México, donde la salinidad y la temperatura son dos de los factores importantes a considerar en el cultivo de esta especie y de otros peneidos (1). Sin embargo, cuando las postlarvas son comercializadas por los laboratorios de producción a las granjas de camarón, generalmente las postlarvas son distribuidas a diferentes condiciones de salinidad y temperatura dependiendo de su ubicación geográfica de las granjas camaronícolas.

Por ello, es importante que las postlarvas de camarón antes de ser sembradas en los estanques, sean sometidas a diferentes condiciones de salinidad y temperatura para estimar previamente su calidad en la supervivencia (2). La prueba de salinidad y temperatura utilizada en el presente estudio para postlarvas de camarón blanco, es simple, rápida y no requiere de equipo especializado $(1,3)$. Es una prueba recomendable para los laboratorios de producción comercial o granjas de camarón para evaluar la condición fisiológica de las postlarvas en cuanto a su supervivencia, aunque no necesariamente determine su éxito en su crecimiento y biomasa cuando han sido sembradas después en los estanques de engorda de camarón.

Algunos autores han analizado los criterios para asignar una calidad en la supervivencia de larvas y postlarvas de $L$. vannamei, para lo cual es necesario que se emplee simultáneamente la salinidad y temperatura (4). Otros investigadores han considerado que la temperatura no es un factor importante en la supervivencia de postlarvas al momento de ser sembradas en los estanques de cultivo, pero han señalado que es más importante someter previamente a las postlarvas a pruebas combinadas de salinidad y amonio $(1,2,5)$. De la misma manera, se estimó en las postlarvas que la salinidad a diferencia de su punto isosmótico, podría ser utilizado como un indicador de la calidad de las mismas, incluso, estos autores no consideraron como un indicador a la temperatura (6). Sin embargo, se ha indicado que una adecuada alimentación en los primeros estadios larvales en camarón blanco, han favorecido al crecimiento, supervivencia $(7,8)$ y su resistencia a los cambios de salinidad y temperatura (9). Además, se ha indicado la necesidad de estandarizar la metodología para evaluar la calidad de las postlarvas ante las diversas pruebas mencionadas, aunque para ello debe considerarse tanto la salinidad como la temperatura (10).

Existen informes donde la salinidad y la temperatura en conjunto con otras variables hidrológicas, han determinado la influencia fundamental en la supervivencia y en el desarrollo de larvas y postlarvas de camarones peneidos (11-13), particularmente sometidas a pruebas de estrés $(1,3,9)$.

Algunos autores, han indicado que las condiciones óptimas para la supervivencia de postlarvas de L. stylirostris correspondió a 25 ups y $28^{\circ} \mathrm{C}$ (14). En $L$. vannamei determinaron que la temperatura 
adecuada para la supervivencia de postlarvas resultó entre 26.1 y $31.4^{\circ} \mathrm{C}$ (15). Mientras que otros señalaron que la supervivencia de postlarvas de camarón blanco resultó significativa a la concentración de salinidad (16), contrariamente otros indicaron que las salinidades no influyeron directamente en la supervivencia de postlarvas (17).

Además, diversas investigaciones han señalado que el potencial de producción y la capacidad de carga biológica de un sistema de cultivo son generalmente determinados en conjunto por las condiciones del ambiente acuático, las cuales pueden alterar las tasas de actividad biológica y por consecuencia modificar el crecimiento de las larvas y postlarvas y en algunos casos pérdidas irreparables provocando su mortalidad. Entonces, es necesario analizar las condiciones de supervivencia de las postlarvas de L. vannamei a diferentes salinidades y temperaturas, ya que pueden ser indicativos para su evaluación del estado fisiológico de las postlarvas y por lo tanto predecir el efecto causado por las variaciones de salinidad y temperatura, ya que estos, entre otros, son los factores abióticos dominantes en los sistemas acuícolas.

Como objetivo principal se analizó la supervivencia de postlarvas de L. vannamei sometidas a diferentes salinidades y temperaturas. Sobre todo, para poder determinar así la mejor condición de las postlarvas de camarón blanco ante estas variables a las cuales generalmente son sometidas las postlarvas durante su siembra en las diferentes granjas comerciales del país.

\section{MATERIALES Y MÉTODOS}

Postlarvas y salinidad del agua. Se utilizaron postlarvas (PL12 -doce días en fase de postlarva-) de camarón blanco ( $L$. vannamei) con un peso seco (PS) promedio de $0.20 \mathrm{mg}$ proporcionadas por un laboratorio de producción comercial (FITMAR) del sur de Sinaloa, México. Las postalrvas se transportaron al laboratorio en una hielera dentro de bolsas de plástico con agua a saturación de oxígeno y a temperaturas de 26 a $28^{\circ} \mathrm{C}$. Las postlarvas a las mismas condiciones de salinidad y temperatura de su procedencia, se colocaron en un tanque de plástico con una capacidad de $400 \mathrm{~L}$ con agua de mar filtrada a 10,5 y $1 \mu \mathrm{m}$ y se adicionó aireación continua con un soplador (blower de 2.5 HP). Posteriormente, se mantuvieron las postlarvas con recambios diarios de $30 \%$, la alimentación previa a los experimentos fue cada dos horas en forma de "microparticulado" de tipo Frippak PL+300 y Stresspack 1.2.3. La eliminación de heces y del alimento sobrante, se realizó por un sifón protegido con una malla nytex de $200 \mu \mathrm{m}$.

El oxígeno disuelto en los tratamientos fue de 6.95 a $9.71 \mathrm{mg} / \mathrm{L}$ y la concentración de amonio varío de 0.0002 y $0.005 \mathrm{mg} / \mathrm{L} / \mathrm{L} / \mathrm{hPS}$. Para determinar la supervivencia de postlarvas, se realizó un experimento en combinación con cinco salinidades $(5,15,25,35$ y 45ups) y cinco temperaturas $\left(15,20,25,30\right.$ y $\left.35^{\circ} \mathrm{C}\right)$. Para incrementar la salinidad (45ups), se añadió sal granulada (sin yodo) al agua de mar a una concentración de $10 \mathrm{~g} / \mathrm{L}$, para disminuir la salinidad, se agregó agua dulce filtrada a 10, 5 y $1 \mu \mathrm{m}$ (filtro de cartucho) al agua de mar, después se determinó la concentración de salinidad en los tratamientos con un refractómetro digital VITALSINE, SR-6.

Diseño experimental. Se utilizaron acuarios de plástico con una capacidad de 4 I, aunque las postlarvas se mantuvieron en $3 \mathrm{~L}$ a una densidad de 100 postlarvas/L. Por cada salinidad $(5,15,25,35$ y 45 ups $)$ se utilizaron 20 acuarios distribuidos en cuatro repeticiones. Para mantener la temperatura del agua constante en los experimentos $\left(15,20,25,30\right.$ y $\left.35^{\circ} \mathrm{C}\right)$, cada acuario contenía un calentador y un termorregulador FINNER, HC-0800.

Para estimar la supervivencia y frecuencia de mudas, se filtraron las postlarvas en la salinidad inicial (35ups), y se mantuvieron en un recambio de agua del $100 \%$ con la salinidad deseada (5, 15, 25, 35 y 45ups) durante 30 minutos. Posteriormente, las postlarvas en las diferentes salinidades se filtraron de nuevo y se regresaron a su condición salina inicial (35ups) por 30 minutos; posteriormente a este tiempo transcurrido se contaron las postlarvas vivas y las mudas, luego se registraron los porcentajes (\%) de supervivencia (porcentaje de organismos vivos al final del experimento con respecto al inicio) y frecuencia de mudas (porcentaje de organismos muertos y frecuencia de mudas a diferencia del inicial).

Análisis estadístico. Los datos del porcentaje de supervivencia y en conjunto de mudas y mortalidad, fueron trasformados a arcoseno para su análisis estadístico. A los datos trasformados de supervivencia y mudas y mortalidad por cada combinación de salinidad y temperatura se les realizaron pruebas estadísticas de normalidad (Lillieford) y homoscedasticidad (Bartlett). Posteriormente se analizaron por un ANOVA de dos vías, como tratamientos la supervivencia y frecuencia de mudas, y como factor A, la salinidad (ups) y B, la temperatura $\left({ }^{\circ} \mathrm{C}\right)$. Al resultar significativo el ANOVA de dos 
vías, se realizaron pruebas de comparaciones múltiples de Tukey; en todos los casos se utilizó un nivel de significancia (a) de 0.05 (18) y el ajuste de medias se calculó por el paquete Statistica, V 7.0 (19). Sin embargo, los datos de la supervivencia y mudas y mortalidad fueron trasformados nuevamente a sus porcentajes para su interpretación comparativa de los datos.

\section{RESULTADOS}

Los porcentajes de supervivencia de postlarvas durante las diferentes combinaciones de salinidad y temperatura, oscilaron en valores altos de 94.4 y $99.8 \%$, pudiendo ser consideradas las postlarvas de calidad para su posterior siembra en estanques de cultivo. En general, los menores porcentajes de supervivencia se observaron a una salinidad de 5 ups en combinación con temperaturas de 15,30 y $35^{\circ} \mathrm{C}$. Aunque, la mayor mortalidad de postlarvas coincidió con una salinidad y temperatura de 5 ups y $30^{\circ} \mathrm{C}$, respectivamente.

La supervivencia, estadísticamente resultó significativa con respecto a la salinidad, temperatura y su interacción $(p<0.05)$. Estos resultados indicaron que la supervivencia de postlarvas de camarón blanco dependieron de las condiciones de salinidad, temperatura y de la interacción de ambos factores hidrológicos a los cuales fueron sometidas las postlarvas (Tabla 1). Lo que confirmó que las postlarvas de $L$. vananmei aún y cuando generalmente se caracterizan por su amplia tolerancia a una variación salina y térmica, el efecto de dichas variables y de su interacción, están relacionadas directamente con el éxito de la supervivencia de las postlarvas debido a que en promedio se obtuvo un porcentaje de supervivencia de $98.7 \%$ entre las diferentes combinaciones de salinidad y temperatura.

En particular, los promedios de la supervivencia de postlarvas presentaron una significancia entre los tratamientos de 5 ups y $15^{\circ} \mathrm{C}$ con el resto

Tabla 1. ANOVA para la supervivencia de postlarvas sometidas a diferentes salinidades (ups) y temperaturas $\left({ }^{\circ} \mathrm{C}\right)$.

\begin{tabular}{lcccccc}
\hline \multicolumn{1}{c}{ FV } & SC & GL & CM & F & P & F \\
\hline Salinidad (ups) & 1021.0 & 4 & 255.3 & 30.3 & $3.7 \times 10^{-19}$ & 2.4 \\
Temperatura $\left({ }^{\circ} \mathrm{C}\right)$ & 342.6 & 4 & 85.7 & 10.2 & $2.0 \times 10^{-7}$ & 2.4 \\
Interacción & 541.8 & 16 & 33.9 & 4.0 & $1.7 \times 10^{-6}$ & 1.7 \\
Residual & 1474.5 & 175 & 8.4 & & & \\
Total & 3379.8 & 199 & & & & \\
\hline
\end{tabular}

FV: Fuente de variación, SC: suma de cuadrados, GL: grados de libertad, CM: cuadrados medios, F: estadístico, P: probabilidad. de las salinidades utilizadas. A 30 y $35^{\circ} \mathrm{C}$ entre las salinidades de 5 y 45 ups, no se encontraron diferencias estadísticas entre los porcentajes de supervivencia, pero a 45 ups comparado con 15 , 25 y 35ups, la supervivencia de postlarvas no resultó con diferencias para dichas temperaturas mencionadas (Figura 1).

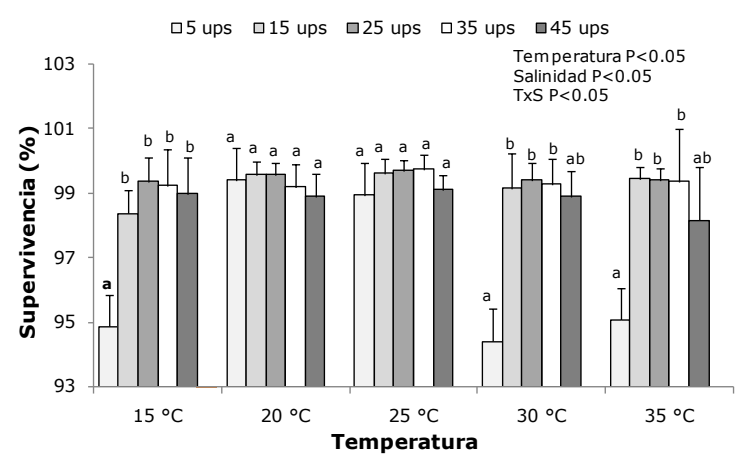

Figura 1. Supervivencia y desviación estándar de postlarvas sometidas a diferentes salinidades y temperaturas. Letras distintas indican diferencias significativas.

Los menores porcentajes de la resistencia (frecuencia de mudas y mortalidad) a los cambios de salinidad y temperatura se ubicaron a una salinidad de 5ups con temperaturas de 15, 30 y $35^{\circ} \mathrm{C}$, correspondiendo a $7.3,6.5$ y $5.5 \%$, respectivamente. Al igual que la supervivencia, los resultados en conjunto de la frecuencia de mudas y postlarvas muertas (Tabla 2 ), fueron afectados por la salinidad, temperatura y su interacción $(p<0.05)$. Sin embargo, cuando se analizaron los datos entre los tratamientos, resultó que a una temperatura de 20 y $25^{\circ} \mathrm{C}$ la frecuencia de mudas y la mortalidad de las postlarvas no resultaron con diferencias estadísticas entre las salinidades utilizadas (Figura 2).

En general se consideró que bajo estas combinaciones de salinidad y temperatura a las cuales fueron expuestas las postlarvas de $L$. vannamei, presentaron condiciones favorables

Tabla 2. ANOVA para la frecuencia de mudas y mortalidad de postlarvas sometidas a diferentes salinidades (ups) y temperaturas $\left({ }^{\circ} \mathrm{C}\right.$ ).

\begin{tabular}{lcccccc}
\hline \multicolumn{1}{c}{ FV } & SC & GL & CM & F & P & F \\
\hline Salinidad (ups) & 312.1 & 4 & 78.0 & 26.8 & $2.4 \times 10^{-17}$ & 2.4 \\
Temperatura $\left({ }^{\circ} \mathrm{C}\right)$ & 115.7 & 4 & 28.9 & 9.9 & $2.9 \times 10^{-7}$ & 2.4 \\
Interacción & 182.6 & 16 & 11.4 & 3.9 & $2.8 \times 10^{-6}$ & 1.7 \\
Residual & 509.8 & 175 & 2.9 & & & \\
Total & 1120.2 & 199 & & & & \\
\hline
\end{tabular}

FV: Fuente de variación, SC: suma de cuadrados, GL: grados de libertad, CM: cuadrados medios, F: estadístico, P: probabilidad. 


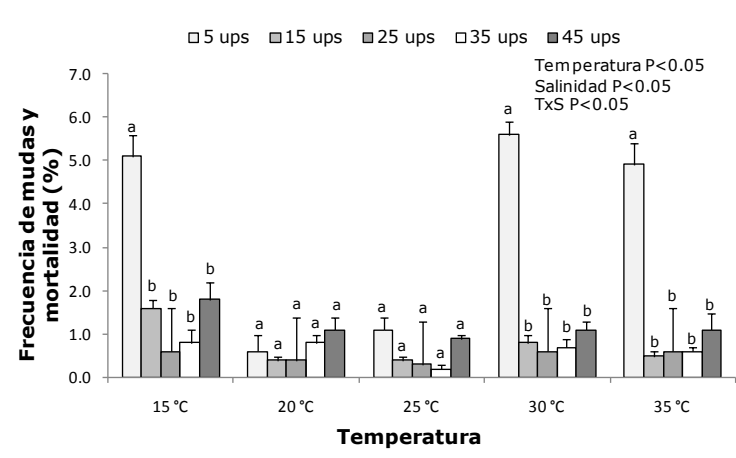

Figura 2. Frecuencia de mudas y mortalidad y desviación estándar de postlarvas sometidas a diferentes salinidades y temperaturas. Letras distintas indican diferencias significativas.

de supervivencia y de resistencia al efecto provocado por estas variables hidrológicas. Aunque en condiciones de temperatura de 20 y $25^{\circ} \mathrm{C}$ en combinación con cualquier salinidad utilizada en el presente estudio, indicaron para las postlarvas los mejores porcentajes de supervivencia y de resistencia a los efectos de la salinidad.

\section{DISCUSIÓN}

Para los acuicultores la supervivencia es un importante indicador para la toma de decisiones relacionadas con la modificación de las condiciones de cultivo y para la producción de postlarvas, por lo tanto, entre otros indicadores, la supervivencia refleja las condiciones de éxito o fracaso en las que los organismos son cultivados. Altas supervivencias están directamente relacionadas con condiciones apropiadas de mantenimiento, sin embargo, algunos investigadores analizaron el efecto combinado de la salinidad y temperatura en postlarvas de peneidos y concluyeron que la respiración, excreción y crecimiento en biomasa representaron mejores indicadores que la supervivencia $(3,9,10)$. Aunque, se ha determinado que la salinidad y temperatura presentaron un efecto positivo en las supervivencias de postlarvas de L. vannamei y L. stylirostris $(6,16,20)$.

En el presente estudio, los elevados porcentajes de supervivencia alcanzados independientemente de las diferentes combinaciones de salinidad y temperatura, indicaron que bajo estas condiciones se garantiza la supervivencia óptima de postlarvas de $L$. vannamei por los laboratorios de producción y granjas de camarón. Sin embargo, es probable que los mayores porcentajes de supervivencia estuvieron relacionados con los efectos de la temperatura sobre el metabolismo de los organismos, debido a que las postlarvas durante el período experimental no fueron alimentadas (20), incluso, algunos autores señalaron la importancia del efecto de la temperatura en la actividad metabólica de las postlarvas de $L$. vannamei y su relación con la supervivencia (21). En este estudio, se observó que las postlarvas por debajo de $20^{\circ} \mathrm{C}$ fueron prácticamente inactivas, por ejemplo, en las postlarvas su desplazamiento se manifestó con un nado débil y errático, no así a $35^{\circ} \mathrm{C}$, donde las postlarvas fueron muy activas sobre la columna de agua.

La supervivencia de postlarvas obtenidas en el presente estudio, incluyendo las más bajas, fueron más altas que las registradas para $L$. vannamei en condiciones de pruebas salinas (9). Además, estos autores indicaron que supervivencias a pruebas salinas por encima de $60 \%$ es considerado indicativo para una buena calidad de postlarvas (22), como los porcentajes aquí registrados.

Algunos autores han señalado que la salinidad en postlarvas de $L$. vannamei no parece ser un factor crítico entre 1 a 8ups, además, las postlarvas han soportando comúnmente condiciones hasta los 35ups $(10,13)$. Sin embargo, indicaron que a una mayor salinidad, la supervivencia de postlarvas disminuye (21). En el presente trabajo no se probó una salinidad menor a 5ups, pero sí por arriba de 35ups, aunque en general los resultados de la supervivencia obtenidos entre las diferentes combinaciones de salinidad y temperatura se ubicaron por encima de $94.4 \%$, por lo tanto, las postlarvas sometidas a todas las salinidades y temperaturas que sobrevivieron, a excepción del tratamiento de 5ups con $15^{\circ} \mathrm{C}$, se consideraron como postlarvas de calidad (3), asumiendo que presentaron condiciones fisiológicas (supervivencia) para soportar la tolerancia a los cambios de salinidad y temperatura a las cuales fueron colocadas experimentalmente.

Es probable que los porcentajes mínimos de frecuencia de mudas y mortalidad provocado en las postlarvas estén relacionados con el tiempo de exposición, debido a que algunos autores han señalado que tanto la edad de las postlarvas como el tiempo de exposición a los cambios de salinidad y temperatura, influyeron en el éxito de su resistencia, determinando así porcentajes de supervivencia menores al 50\% cuando fueron sometidas a un tiempo mayor de exposición para diferentes postlarvas de peneidos $(9,23,24)$, a diferencia del presente estudio donde se utilizó un menor tiempo (30 min) en las postlarvas expuestas al cambio de salinidad y temperatura. Los porcentajes de la mortalidad 
fueron similares a otras investigaciones al determinar en postlarvas de camarón blanco una mayor mortalidad en salinidades de 30 y 35 ups con temperaturas de 30 y $35^{\circ} \mathrm{C}(21)$, además estos autores indicaron una elevada mortalidad (73\%) de postlarvas en condiciones de salinidad de 50 ups y temperatura de $35^{\circ} \mathrm{C}$.

Se observó que las postlarvas al ser sometidas a los cambios drásticos de salinidad de 35 a $45,25,15$ y 5 ups en todas las temperaturas utilizadas, aparentemente las postlarvas se dirigieron al fondo de los acuarios, sin mostrar ningún movimiento de apéndices, por lo que se consideraron como postlarvas muertas por algunos autores (3). Sin embargo, en el presente estudio esta "mortalidad" fue aparente, ya que las postlarvas se recuperaron a través del tiempo, sobre todo cuando se regresaron a la salinidad inicial (35ups). Incluso en este estudio se detectaron entre los tratamientos supervivencias altas después de su recuperación, considerándolas como postlarvas de calidad de acuerdo con los criterios establecidos para $L$. vannamei (1).

Los porcentajes de supervivencia obtenidos, confirmaron que a salinidades entre 5 y 45 ups en combinación con temperaturas de 20 y $25^{\circ} \mathrm{C}$, las postlarvas de camarón blanco soportaron una amplia variación salina, pero en condiciones de temperatura de 15,30 y $35^{\circ} \mathrm{C}$ con una salinidad de 5ups, disminuyeron los porcentajes de supervivencia de postlarvas; sobre todo cuando no se cumplieran con los protocolos de producción de calidad de larvas y postlarvas (1), principalmente en cuanto a su selección de sus reproductores y del suministro del alimento en cantidad y calidad adecuada para el mantenimiento de las postlarvas. Los resultados de los porcentajes altos de supervivencias obtenidas en el presente estudio, son debido a que $L$. vannamei es una especie eurihalina que en condiciones naturales puede sobrevivir a una amplia variación salina $(1,4)$, soportando así las postlarvas las salinidades entre 5 y 45 ups a las cuales fueron expuestas.

Por otro lado, en el caso del tiempo de exposición ante los cambios salino y térmico sea considerado relativamente corto y el análisis de la supervivencia indicó que se presentaron diferencias significativas entre los tratamientos de la salinidad, temperatura y su interacción, es conveniente recomendar la utilización de otros indicadores que permitan evaluar un mayor efecto de los tratamientos en las postlarvas de $L$. vannamei. Con este propósito, se han empleado pruebas que midieron la resistencia de las postlarvas a diferentes condiciones de salinidad y temperatura sobre el consumo de oxígeno, excreción de amonio y su crecimiento $(3,5,22)$, de tal forma que los resultados fueron expresados generalmente a través de índices que reflejaron una mejor interpretación de la supervivencia de las postlarvas. En este estudio, es probable que diversos factores hayan influido sobre la supervivencia obtenida (combinación de baja salinidad y baja o alta temperatura), ya que la utilización de una sola variable (PL12) determinó que las similitudes o diferencias encontradas en los tratamientos ante las diferentes combinaciones de temperatura y salinidad, se debió al efecto de estas variables hidrológicas únicamente sobre el estadio (PL12), a diferencia de la utilización de diferentes estadios de postlarvas (23). Aunque existen evidencias de que postlarvas más jóvenes a PL12, son menos resistentes a los cambios drásticos de salinidad y temperatura, debido principalmente a que sus branquias no se han desarrollado completamente $(23,24)$.

En conclusión se determinó que a temperaturas de 20 y $25^{\circ} \mathrm{C}$ en combinación con las salinidades utilizadas, las condiciones de supervivencia de postlarvas fueron mejores, lo cual coincidió con las características de la mayoría de los laboratorios de producción de postlarvas comerciales de L. vannamei ubicados cercanamente a aguas costeras del noroeste de México. Además, que estos valores de salinidad y temperatura, se encontraron dentro de los señalamientos adecuados para una supervivencia de postlarvas de camarón blanco ante las diferentes combinaciones de salinidad y temperatura a las cuales generalmente son sometidas a siembras en granjas comerciales del noroeste del país (25).

\section{Agradecimientos}

A los proyectos UAS-CA-162, PROFAPI-135-2009 por los recursos otorgados, al CONACYT por la beca asignada al primer autor (CVU 256237), al personal del Laboratorio de Producción de Postlarvas FITMAR, a Martin Alejandro Guerrero Ibarra por la recolección de datos y Ph.D Miguel Ángel Hurtado Oliva por sus comentarios al manuscrito. 


\section{REFERENCIAS}

1. Racotta I, Palacios E, Hernández R, Bonilla A, Pérez C, Ramírez JL. Criteria for assessing larval and postlarval quality of Pacific white shrimp (Litopenaeus vannamei). Aquacult 2004; 233:181-195.

2. Arzola GJ, Flores CL, Izabal CA, Gutiérrez RY. Crecimiento de camarón blanco (Litopenaeus vannamei) en un estanque rústico de baja salinidad. Rev Aquatic 2008; 28:8-15.

3. Palacios E, Racotta IS. Salinity stress test and its relation to future performance and different physiological responses in shrimp postlarvae. Aquacult 2007; 233:123-135.

4. Martínez CL. Camaronicultura avances y tendencias. México: AGT Editor: 2002.

5. Barbieri E. Acute toxicity of ammonia in white shrimp (Litopenaeus schmitti) at different salinity levels. Aquacult 2010; 306:329-333.

6. Alvarez AL, Racotta IS, Arjona O, Palacios E. Salinity stress test as a predictor of survival during growout in Pacific white shrimp (Litopenaeus vannamei). Aquacult 2004; 237:237-249.

7. Piña VP, Nieves SM, Ramos L, Chavira CO, Voltolina D. Survival, growth and feeding efficiency of Litopenaeus vannamei protozoea larvae, fed different rations of diatom Chaetoceros muelleri. Aquacult 2005; 249:431-437.

8. Piña VP, Voltolina $D$, Nieves SM, Robles $M$. Survival, development and growth of the Pacific white shrimp Litopenaeus vannamei protozoea larvae, fed with monoalgal and mixed diets. Aquacult 2006; 253:523-530.

9. Gelabert R, Brito R, Gaxiola MG, Castro T, Rosas C. Efecto de nauplios de Artemia franciscana enriquecidos sobre el crecimiento, supervivencia y resistencia al estrés de postlarvas (PL5-40) de Litopenaeus vannamei. Univ Cien 2008; 24:29-40.

10. Valenzuela W, Rodríguez G, Ponce PJ, Esparza $\mathrm{H}$. Efecto de diferentes combinaciones de temperatura y salinidad sobre el consumo específico de oxígeno en el camarón blanco Litopenaeus vannamei. Rev Biol Mar y Oceanog 2011; 46:303-311.
11. Díaz F, Re AD, Sierra E, Diaz, IE. Effects of temperature and salinity fluctuation on the oxygen consumption, ammonium excretion and osmoregulation of the blue shrimp Litopenaeus stylirostris. J Shellfish Res 2004; 23:903-910.

12. Ramos CS, Ramos SE. Abundancia relativa de postlarvas de camarones peneidos en la bahía Salinas del Marqués, Golfo de Tehuantepec, México. Marzo a junio de 1999. Rev Biol Mar y Oceanog 2006; 41:121-128.

13. Valenzuela W, Rodríguez G, Esparza H. Cultivo intensivo de camarón blanco Litopenaeus vannamei en agua de pozo a baja salinidad como alternativa acuícola en zonas de alta marginación. Ra Ximhai 2010; 6:1-8.

14. Re AD, Díaz F, Sierra E, Gómez S. Consumo de oxígeno, excreción de amonio y capacidad osmorreguladora de Litopenaeus stylirostris expuesto a diferentes combinaciones de temperatura y salinidad. Ciencias Marinas, 2004; 30(3):443-453.

15. Hernández RM, Bückle LF, Palacios E, Barón SB. Preferential behavior of white shrimp Litopenaeus vannamei (Boone 1931) by progressive temperature-salnity simultaneous interaction. J Therm Biol 2006; 31(7):565-572.

16. Jaime B, Galindo J, Laria E, Cupul F, Vega F. Traslado de postlarvas de Litopenaeus vannamei a diferentes tiempos, salinidades y densidades y su efecto en la supervivencia y algunos marcadores bioquímicos. Rev Biol Mar Oceanog 2008; 43:681-686.

17. Ye $L$, Jiang $S$, Zhu $X$, Yang $Q$, Wen $W$, Wu $K$. Effects of salinity on growth and energy budget of juvenile Penaeus monodon. Aquacult 2009; 290:140-144.

18. Zar JH. Biostatistical analysis. Upper Saddle River, USA: Prentice-Hall Inc; 2009.

19. StatSoft Inc. Statistica for Windows Manual version 7.0. Tulsa, Oklahoma, USA: StatSoft Inc; 2004. 
20. Spanopoulus M, Martínez CA, Vanegas RC, Rosas C, Ross LG. The combined effects of salinity and temperature on the oxygen consumption of juvenile shrimps Litopenaeus stylirostris. Aquacult 2005; 244:341-348.

21. Ponce J, Martínez CA, Ross LG. The effects of salinity and temperature on the growth and survival rates of juvenile White shrimp, Penaeus vannamei. Aquacult 1997; 157:107-115.

22. Barbieri E. Use of oxygen consumption and ammonium excretion to evaluate the sublethal toxicity of cadmium and zinc on Litopenaeus schmitti. Water Environ Res 2007; 79:461-466.
23. Balbi F, Rosas J, Velásquez A, Cabrera T, Maneiro C. Aclimatación de postlarvas de diferentes edades y criaderos de camarón marino Litopenaeus vannamei. Rev Biol Mar y Oceanog 2005; 40:109-115.

24. Tsuzuki M, Cavalli RO, Bianchini A. The effects of temperature, age and acclimation to salinity on the survival of Farfantepenaeus paulensis postlarvae. J World Aquacult Soc 2000; 31:459-468.

25. Martínez CL, Martínez PM, Cortes JE. Camaronicultura mexicana y mundial cactividad sustentable o industria contaminante?. Rev Int Contam Ambie 2009; 25:181-196. 